November 4, 2018

hep-ph/0101299

\title{
Precision Physics Near LEP Shutdown and Evolutionary Developments
}

\author{
GIAMPIERO PASSARINO用 \\ Dipartimento di Fisica Teorica, Università di Torino, Italy \\ INFN, Sezione di Torino, Italy
}

\begin{abstract}
The most celebrated aspects of precision physics are briefly summarized. New ideas are also introduced that may lead to further developments in the field of radiative corrections, with special emphasis to multiloop numerical evaluation and to a correct treatment of QED radiation for arbitrary processes.
\end{abstract}

Presented at the

50 Years of Electroweak Physics

A symposium in honor of Professor

Alberto Sirlin's 70th Birthday

October 27-28, 2000

*Work supported by the European Union under contract HPRN-CT-2000-00149. 


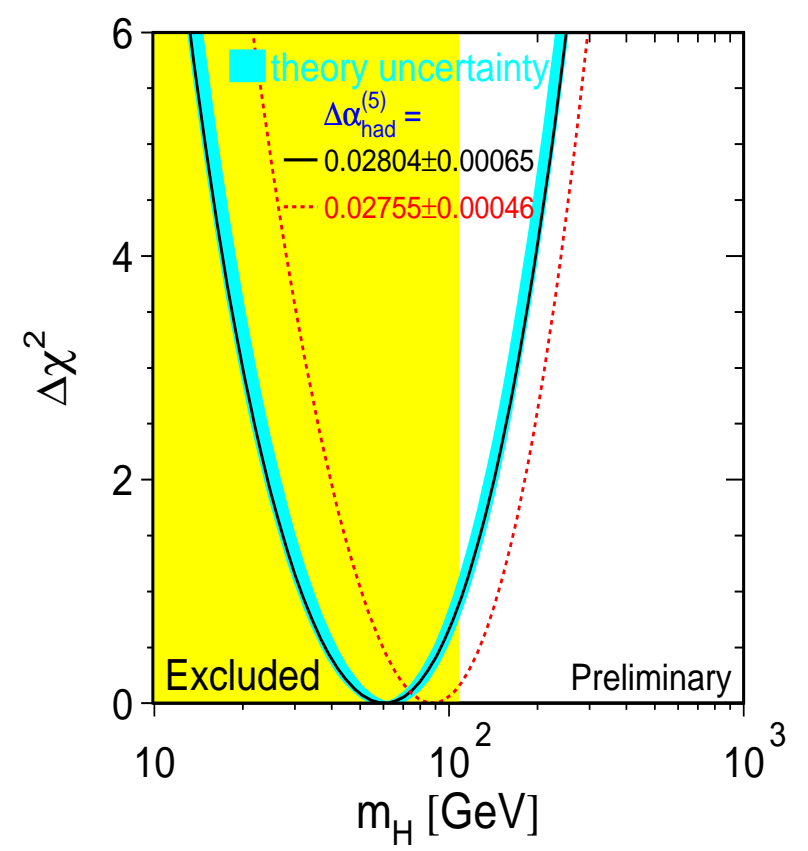

\section{The experimental progress}

The experimental progress made in the 1990's is remarkable in many ways. With the right experiments we can reveal the fine details of high energy phenomena. On the eve of LEP shutdown we known that physics at the microscopic scale has a basis as rationale as chemistry, everything moves, not by magic, but because there is a mechanism at work. Everything is encouraging for the major unsolved aspects of the breaking of electroweak gauge symmetry.

The blue band, giving the theoretical uncertainty in the $\chi^{2}$-distribution as a function of the Higgs boson mass is, perhaps, the most celebrated figure of the whole LEP era. It is interesting to examine the environment in which the technology for producing this figure has been developed. One way is through the Kühn tower. According to this dantescan representation we find at the top of the tower a group of highlyspecialized experts: QED, QCD etc. Next to them we will find the Smugglers: those who assemble a code mooncursing it. At the lower level the Users are represented: the experimental community at large.

The Rosetta stone that keeps this world running is based on some sort of universal language that has been created to allow for a meaningful communication, the PseudoObservable language.

\subsection{The language of Pseudo-Observables}

The PO are related to measured cross-sections and asymmetries by some deconvolution or unfolding procedure and the concept itself of pseudo-observability is rather 


\begin{tabular}{|c|c|c|c|}
\hline \multicolumn{4}{|c|}{ Osaka 2000} \\
\hline & Measurement & Pull & \begin{tabular}{llllll} 
& \multicolumn{5}{c}{ Pull } \\
-2 & -1 & 0 & 1 & 2 & 3
\end{tabular} \\
\hline $\mathrm{m}_{\mathrm{z}}[\mathrm{GeV}]$ & $91.1875 \pm 0.0021$ & .05 & \\
\hline$\Gamma_{Z}[\mathrm{GeV}]$ & $2.4952 \pm 0.0023$ & -.42 & - \\
\hline$\sigma_{\text {hadr }}^{0}[\mathrm{nb}]$ & $41.540 \pm 0.037$ & 1.62 & \\
\hline$R_{1}$ & $20.767 \pm 0.025$ & 1.07 & 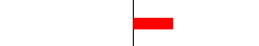 \\
\hline$A_{\mathrm{fb}}^{0,1}$ & $0.01714 \pm 0.00095$ & .75 & 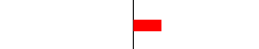 \\
\hline$A_{e}$ & $0.1498 \pm 0.0048$ & .38 & $F$ \\
\hline$A_{\tau}$ & $0.1439 \pm 0.0042$ & -.97 & \\
\hline $\sin ^{2} \theta_{\text {eff }}^{\text {lept }}$ & $0.2321 \pm 0.0010$ & .70 & 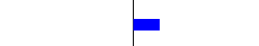 \\
\hline $\mathrm{m}_{\mathrm{W}}[\mathrm{GeV}]$ & $80.427 \pm 0.046$ & .55 & 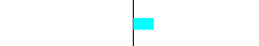 \\
\hline $\mathrm{R}_{\mathrm{b}}$ & $0.21653 \pm 0.00069$ & 1.09 & 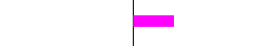 \\
\hline $\mathrm{R}_{\mathrm{c}}$ & $0.1709 \pm 0.0034$ & -.40 & 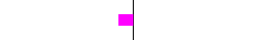 \\
\hline$A_{f b}^{c, b}$ & $0.0990 \pm 0.0020$ & -2.38 & \\
\hline$A_{\mathrm{fb}}^{0, \mathrm{c}}$ & $0.0689 \pm 0.0035$ & -1.51 & \\
\hline$A_{b}$ & $0.922 \pm 0.023$ & -.55 & 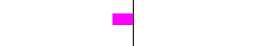 \\
\hline$A_{c}$ & $0.631 \pm 0.026$ & -1.43 & \\
\hline $\sin ^{2} \theta_{\text {eff }}^{\text {lept }}$ & $0.23098 \pm 0.00026$ & -1.61 & \\
\hline $\sin ^{2} \theta_{w}$ & $0.2255 \pm 0.0021$ & 1.20 & ב \\
\hline $\mathrm{m}_{\mathrm{w}}[\mathrm{GeV}]$ & $80.452 \pm 0.062$ & .81 & 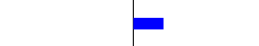 \\
\hline$m_{t}[\mathrm{GeV}]$ & $174.3 \pm 5.1$ & -.01 & \\
\hline$\Delta \alpha_{\text {had }}^{(5)}\left(m_{z}\right)$ & $0.02804 \pm 0.00065$ & -.29 & E \\
\hline
\end{tabular}

difficult to define. One way to introduce it is to say that the experiments measure some primordial (basically cross-sections and thereby asymmetries also) quantities which are then reduced to secondary quantities under some set of specific assumptions. Within these assumptions the secondary quantities, the PO, also deserve the label of observability.

A quick example of this language is as follows. The explicit formulae for the $Z f \bar{f}$ vertex are always written starting from a Born-like form of a pre-factor $\times$ fermionic current, where the Born parameters are promoted to effective, scale-dependent parameters,

$$
\rho_{Z}^{f} \gamma_{\mu}\left[\left(I_{f}^{(3)}+i a_{L}\right) \gamma_{+}-2 Q_{f} \kappa_{Z}^{f} s^{2}+i a_{Q}\right]=\gamma_{\mu}\left(\mathcal{G}_{V}^{f}+\mathcal{G}_{A}^{f} \gamma_{5}\right)
$$

where $\gamma_{+}=1+\gamma_{5}$ and $a_{Q, L}$ are the SM imaginary parts.

By definition, the total and partial widths of the $Z$ boson include also QED and QCD corrections. The partial decay width is therefore described by the following expression:

$$
\Gamma_{f} \equiv \Gamma(Z \rightarrow f \bar{f})=4 c f . \Gamma_{0}\left(\left|\mathcal{G}_{V}^{f}\right|^{2} R_{V}^{f}+\left|\mathcal{G}_{A}^{f}\right|^{2} R_{A}^{f}\right)+\Delta_{\mathrm{EW} / \mathrm{QCD}}
$$

where $c f$. $=1$ or 3 for leptons or quarks $(f=l, q)$, and $R_{V}^{f}$ and $R_{A}^{f}$ describe the final state QED and QCD corrections and take into account the fermion mass $m_{f}$. The last term,

$$
\Delta_{\mathrm{EW} / \mathrm{QCD}}=\Gamma_{\mathrm{EW} / \mathrm{QCD}}^{(2)}-\frac{\alpha_{S}}{\pi} \Gamma_{\mathrm{EW}}^{(1)}
$$


accounts for the non-factorizable corrections. The standard partial width, $\Gamma_{0}$, is

$$
\Gamma_{0}=\frac{G_{F} M_{Z}^{3}}{24 \sqrt{2} \pi}=82.945(7) \mathrm{MeV}
$$

The peak hadronic and leptonic cross-sections are defined by

$$
\sigma_{h}^{0}=12 \pi \frac{\Gamma_{e} \Gamma_{h}}{M_{Z}^{2} \Gamma_{Z}^{2}} \quad \sigma_{\ell}^{0}=12 \pi \frac{\Gamma_{e} \Gamma_{l}}{M_{Z}^{2} \Gamma_{Z}^{2}},
$$

where $\Gamma_{Z}$ is the total decay width of the $Z$ boson, i.e, the sum of all partial decay

widths. The effective electroweak mixing angles (effective sinuses) are always defined by

$$
4\left|Q_{f}\right| \sin ^{2} \theta_{\text {eff }}^{f}=1-\frac{\operatorname{Re} \mathcal{G}_{V}^{f}}{\operatorname{Re} \mathcal{G}_{A}^{f}}=1-\frac{g_{V}^{f}}{g_{A}^{f}}, \quad g_{V}^{f}=\operatorname{Re} \mathcal{G}_{V}^{f}, \quad g_{A}^{f}=\operatorname{Re} \mathcal{G}_{A}^{f} .
$$

The forward-backward asymmetry $A_{\mathrm{FB}}$ is defined via

$$
A_{\mathrm{FB}}=\frac{\sigma_{\mathrm{F}}-\sigma_{\mathrm{B}}}{\sigma_{\mathrm{F}}+\sigma_{\mathrm{B}}}, \quad \sigma_{\mathrm{T}}=\sigma_{\mathrm{F}}+\sigma_{\mathrm{B}}
$$

where $\sigma_{\mathrm{F}}$ and $\sigma_{\mathrm{B}}$ are the cross-sections for forward and backward scattering, respectively.

\section{A new era}

Admittedly, we are entering a new era: we have been moving from a mediaeval architecture of radiative corrections to the renaissance of LEP period and now one should not indulge in deciphering the intimate meaning of the last digit in the last fit of the LEPEWWG.

A new frontier is at the horizon: most likely it is goodbye to the one man show. Running a new RC project will be a little like running an experiment.

At the eve of LEP shutdown it is of some importance to summarize the present status of high precision physics [1]. For $e^{+} e^{-} \rightarrow \bar{f} f$ all one-loop terms are known, including re-summation of leading terms. At the two-loop level leading and nextto-leading terms have been computed and included in codes like TOPAZ0 [2,3] and ZFITTER [4]. For realistic observables initial state QED radiation is included via the structure function method, or equivalent ones. Final state QED is also available as well as the interference between initial and final states [5]. Fine points in QED for $2 \rightarrow 2$ are as follows. For $s$-channel all the $\mathcal{O}\left(\alpha^{2} L^{n}\right), n=0,1,2$ terms are known from explicit calculations, the leading $\mathcal{O}\left(\alpha^{3} L^{3}\right)$ is also available and they are important for the studies of the $Z$ lineshape. 
Differences and uncertainties amount to at most $\pm 0.1 \mathrm{MeV}$ on $M_{Z}$ and $\Gamma_{Z}$ and $\pm 0.01 \%$ on $\sigma_{\mathrm{h}}^{0}$ (MIZA, TOPAZ0 and ZFITTER) [6] For non-annihilation processes (Bhabha) both structure-function and parton-shower methods have been analyzed and the uncertainty is estimated to be $0.061 \%$ from BHLUMI [7]. Certainly, full two-loop electroweak corrections are needed for GigaZ $\left(10^{9} Z\right.$ events) with a quest for a fast numerical evaluation of the relevant diagrams.

For $e^{+} e^{-} \rightarrow 4$ fermions all tree-level processes are available and $\mathcal{O}(\alpha)$ electroweak corrections are known only for the $W W$-signal and in double-pole approximation (DPA) 8] and [9]. $e^{+} e^{-} \rightarrow 4 \mathrm{f}+\gamma$ in Born approximation is also available for all processes [10]. Dominant radiative corrections are inserted in single- $W$ production through the so-called Fermion-Loop scheme [11]13].

Fine points in QED for $2 \rightarrow 4$ are as follows For $e^{+} e^{-} \rightarrow W W \rightarrow 4 \mathrm{f}$ DPA gives the answer but, for a generic process $e^{+} e^{-} \rightarrow 4 \mathrm{f}$ QED radiation is included by using $s$-channel structure-functions, i.e. in leading-log approximation. The latter are strictly applicable only if ISR can be separated unambiguously. Otherwise their implementation may lead to an excess of radiation. Preliminar investigations towards non- $s$ SF by GRACE and by SWAP [10] gives an indication on how to implement the bulk of the non-annihilation effect but still represent ad hoc solutions. These methods, which are essentially based on a matching with the soft photon emission, still contain an ambiguity on the energy scale selection with consequences on the predicted observables.

\section{Realistic observables}

How well do we know realistic observables in $e^{+} e^{-} \rightarrow 2 \mathrm{f}$ ? Pretty well around the $Z$ peak, reasonably well up to LEP 2 energies, but fermion-pair corrections (PP) are not really under control. Let us describe the structure of PP by starting with a simple case, $e^{+} e^{-}$PP-corrections to $e^{+} e^{-} \rightarrow \bar{b} b$. PP is more than photon conversion, we will have the following sets of diagrams: Multi-Peripheral, Initial State Singlet (ISS), Initial State Non-Singlet (ISNS) and Final State (FS).

Note that we include both $\gamma$ and $Z$ exchange. On top of real pair production one has to include virtual $e^{+} e^{-}$pairs. Let us compare as a typical example the sum of virtual and real pair corrections for primary hadrons and primary muons at $\sqrt{(} s)=189 \mathrm{GeV}$.

The comparison between GENTLE, ZFITTER, and TOPAZ0 for the diagrambased definition reveals maximum differences of 1.7(1.5) per mill for inclusive hadrons (muons) and 0.2(0.4) per mill for high $s^{\prime}$ hadrons (muons).

However, PP are to be completed expressly at higher energies and above vector boson thresholds. The first evolutionary development is: a $4 \mathrm{f} \mathrm{MC}$ has to be fully interfaced with a $2 \mathrm{f} \mathrm{MC}$. 


\section{QED radiation}

There are several $4 \mathrm{f}$ processes, those with $t$-channel photons that are not dominated by annihilation. Examples are: single- $W$ production and two-photon processes. How to include the bulk of QED radiative corrections? The second evolutionary development: is multi-photon radiation a one-scale or a multi-scale convolution phenomenon?

$$
\sigma\left(p_{+} p_{-} \rightarrow\left\{q_{i}\right\}+\mathrm{QED}\right) \stackrel{?}{=} \int \prod_{\lambda= \pm} d x_{\lambda} D\left(x_{\lambda}, ?\right) \sigma\left(x_{+} p_{+} x_{-} p_{-} \rightarrow\left\{q_{i}\right\}\right)
$$

In the above equation the question mark means that the corresponding scale has to be guessed. We need to understand how the standard SF-method is related to the exact YFS exponentiation. In the standard YFS treatment of multiple photon emission we have

$$
\sigma\left(p_{+}+p_{-} \rightarrow \sum_{i=1,2 l} q_{i}+\sum_{j=1, n} k_{j}\right) \sim \int d P S_{q}\left|M_{0}\right|^{2} E\left(p_{+}+p_{-}-\sum_{i} q_{i}\right)
$$

where $E$ is the spectral function defined through the usual eikonal factor:

$$
\begin{aligned}
E(K) & =\frac{1}{(2 \pi)^{4}} \int d^{4} x \exp (i K \cdot x) E(x) \\
E(x) & =\exp \left\{\frac{\alpha}{2 \pi^{2}} \int d^{4} k e^{i k \cdot x} \delta^{+}\left(k^{2}\right)\left|j^{\mu}(k)\right|^{2}\right\}
\end{aligned}
$$

At this point we choose an alternative procedure were we do not separate the soft component from the hard one and compute some exact result valid for an arbitrary number of dimensions $n$ and for on-shell photons, i.e. $k^{2}=0$,

$$
I=\int d^{n} k e^{i k \cdot x} \frac{\delta^{+}\left(k^{2}\right)}{p_{i} \cdot k p_{j} \cdot k}
$$

In dimensional-regularization one has the following result, valid $\forall x^{2}$ :

$$
I(x)=-\pi \rho \int_{0}^{1} \frac{d u}{P^{2}}\left(\frac{1}{\hat{\varepsilon}}+2 \ln 2-\ln x^{2}-\xi \ln \frac{\xi+1}{\xi-1}\right),
$$

where we have defined a variable $\xi$ as the ratio $\xi=\left|x_{0}\right| / r$, with an infinitesimal imaginary part attributed to $x_{0}$, i.e. $x_{0} \rightarrow x_{0}+i \delta\left(\delta \rightarrow 0_{+}\right)$. Furthermore, $P$ is the linear combination $P=p_{j}+\left(\rho p_{i}-p_{j}\right) u$, where we have defined $\rho$ to satisfy $\left(\rho p_{i}-p_{j}\right)^{2}=0$, and $x_{0}, r$ are rewritten in covariant form as follows:

$$
x_{0}=-\frac{P \cdot x}{\sqrt{-P^{2}}}, \quad r^{2}=x_{0}^{2}+x^{2} .
$$


The last integral shows the infrared pole $\frac{1}{\hat{\varepsilon}}$ and a collection of $\mathrm{Li}_{2}$-functions. Therefore, $E(K)$ is not available in close form. The scheme that we want to propose defines a coplanar approximation [14] to the exact spectral function,

$$
\begin{aligned}
I_{i j}^{c} & \stackrel{\text { def }}{=}-\frac{2}{3} \pi \rho_{i j} \mathcal{F}_{\mathrm{cp}} \frac{1}{p_{j}^{2}-\rho_{i j}^{2} p_{i}^{2}} \ln \frac{\rho_{i j}^{2} p_{i}^{2}}{p_{j}^{2}}, \quad I_{i i}^{c} \stackrel{\text { def }}{=}-\frac{2}{3} \pi \rho_{i j} \mathcal{F}_{\mathrm{cp}} \frac{1}{m_{i}^{2}}, \\
\mathcal{F}_{\mathrm{cp}} & =\ln \left\{e^{-\Delta_{\mathrm{IR}}} \frac{p_{i} \cdot x p_{j} \cdot x}{m_{i} m_{j}}\right\}, \quad \Delta_{\mathrm{IR}}=\frac{1}{\hat{\varepsilon}}+\text { constants. }
\end{aligned}
$$

Within the coplanar approximation we have

$$
\begin{aligned}
E^{\text {pair }<i j>}(K) & \stackrel{\text { cp }}{\rightarrow} \frac{1}{(2 \pi)^{2}}\left\{\frac{e^{-\Delta_{\mathrm{IR}}}}{m_{i} m_{j}}\right\}^{-\alpha A_{i j}} \frac{1}{\Gamma^{2}\left(\alpha A_{i j}\right)} \\
& \times \int_{0}^{\infty} d \sigma d \sigma^{\prime}\left(\sigma \sigma^{\prime}\right)^{\alpha A_{i j}-1} \delta^{4}\left(\sigma p_{i}+\sigma^{\prime} p_{j}-K\right) .
\end{aligned}
$$

This results explains why we have introduced the term coplanar. Note that $\alpha A \sim \beta$ only when the corresponding invariant is much larger than mass ${ }^{2}$ but the above expression is valid for all regimes and it is easily generalized to $n$ emitters with the result that 円 in a process $2 \rightarrow n$ any external charged leg $i$ talks to all other charged legs, each time with a known scale $s_{i j}$ and with a known total weight proportional to

$$
x_{i}^{\alpha\left(A_{1}^{i}+\ldots+A_{I}^{i}\right)-1} / \Gamma\left(\alpha\left(A_{1}^{i}+\ldots+A_{I}^{i}\right)\right), \quad 0 \leq x_{i} \leq 1
$$

Note that each $A$ has the appropriate sign, in/out, part/antp. Furthermore, $I(i)$ is the number of pairs $\langle i j\rangle$ with $i$ fixed. The IR exponent is given by

$$
\alpha A=\frac{2 \alpha}{\pi}\left\{\frac{1+r^{2}}{1-r^{2}} \ln \frac{1}{r}-1\right\}, \quad \frac{m_{e}^{2}}{|t|}=\frac{r}{(1-r)^{2}}
$$

For Bhabha scattering we will have the following combination:

$$
-A\left(s, m_{e}\right)-A\left(t, m_{e}\right)+A\left(u, m_{e}\right)=\frac{2}{\pi}\left[\ln \frac{s t}{m_{e}^{2} u}-1\right],
$$

obtained as an exact result, not a guess.

\subsection{Conclusions for QED}

The structure-function language is still applicable but initial state structure functions evaluated for one scale is, quite obviously, not enough. In any process each

\footnotetext{
${ }^{1}$ A.Ballestrero, G.P. work in progress
} 
external leg brings one structure function; since all charged legs talk to each other, each $\mathrm{SF}$ is not function of one ad hoc scale but all $<i j>$ scales enter into $\mathrm{SF}_{i}$. The exact spectral-function is a convolution of $\mathrm{SF}$

$$
\begin{aligned}
E^{\mathrm{pair}<i j>}(K) & =\int d^{4} K^{\prime} \Phi\left(K^{\prime}\right) E_{\mathrm{cp}}^{\mathrm{pair}<i j>}\left(K-K^{\prime}\right) \\
\Phi(K) & =\frac{1}{(2 \pi)^{4}} \int d^{4} x \exp \left\{i K \cdot x+\alpha\left(I-I_{\mathrm{cp}}\right)\right\}=\delta(K)+\mathcal{O}(\alpha) \cdot(1)
\end{aligned}
$$

Furthermore, IR-finite reminders and virtual parts can be added according to the standard approach of reorganizing the perturbative expansion.

\section{$5 \quad$ Multi-loop calculations}

The third evolutionary development can be summarized as follows. A systematic approach to multi-loop calculations is needed. Although a lot can be done by means of approximations do we have a new, implementable algorithm for a full scale attack to the problem?

There is a new idea around: the Tkachov theorem [15] or generalized Bernstein theorem (see also Bardin's talk).

$$
\mathcal{P}(x, \partial) \prod_{i} V_{i}^{\mu_{i}+1}(x)=B \prod_{i} V_{i}^{\mu_{i}}(x)
$$

where $\mathcal{P}$ is a polynomial of $x=\left(x_{1} \cdots x_{n}\right)$ (a vector of Feynman parameters) and $\partial_{i}=\partial / \partial_{i} ; B$ and all coefficients of $\mathcal{P}$ are polynomials of $\mu_{i}$ and of the coefficients of $V_{i}(x)$.

The message is that we can do numerical calculations. However, one-loop [16] versus multi-loop is exactly as $2 \mathrm{~d}$ Ising model versus $3 \mathrm{~d}$ Ising model. For one-loop we have a universal master formula due to F. V. Tkachov [15]:

$$
\begin{aligned}
V(x) & =x^{t} H x+2 K^{t} x+L, \quad \mathcal{P}=1-\frac{(x+X) \partial_{x}}{2(\mu+1)}, \\
X & =K^{t} H^{-1}, \quad B=L-K^{t} H^{-1} K .
\end{aligned}
$$

An example that everybody can do, the scalar 3-point function

$$
\begin{gathered}
C_{0}=\int_{0}^{1} d x \int_{0}^{x} d y V^{-1-\varepsilon / 2}(x, y)=C_{0}^{d}+C_{0}^{s}+C_{0}^{0}, \\
V(x, y)=a x^{2}+b y^{2}+c x y+d x+e y+f-i \varepsilon \\
C_{0}^{d}=2 \int_{0}^{1} d x \int_{0}^{x} d y V(x, y) \ln V(x, y) G_{2}^{2} \Delta^{-2},
\end{gathered}
$$




$$
\begin{aligned}
& C_{0}^{s}=\frac{1}{2} \int_{0}^{1} d x\left\{V(x, x) \ln V(x, x)\left[G_{2} \Delta^{-2}\left(a_{x}-a_{y}\right)+\frac{3}{2} \Delta^{-1}\left(a_{x}-a_{y}\right) G_{11} \Delta_{1}^{-1}\right]\right. \\
& +V(x, 0) \ln V(x, 0)\left[G_{2} \Delta^{-2} a_{y}+\frac{3}{2} \Delta^{-1} a_{y} G_{12} \Delta_{2}^{-1}\right] \\
& \text { - } \left.V(1, x) \ln V(1, x)\left[G_{2} \Delta^{-2} a_{x}+\frac{3}{2} G_{2} \Delta^{-1} G_{13} \Delta_{3}^{-1}+\frac{1}{2} G_{2}^{2} \Delta^{-2}+\frac{3}{2} \Delta^{-1} a_{x} G_{13} \Delta_{3}^{-1}\right]\right\}, \\
& C_{0}^{0}=-\frac{1}{4} G_{2} \Delta^{-1} a_{3 x} \Delta_{3}^{-1}(b+c+e)-\frac{1}{8} G_{2} \Delta^{-1} G_{13} \Delta_{3}^{-1}\left(\frac{4}{3} b+c+e\right) \\
& +\frac{1}{12} G_{2}^{2} \Delta^{-2}\left(3 a+b+\frac{3}{2} c+4 d+2 e+6 f\right)+\frac{1}{4} \Delta^{-1} a_{x} a_{1 x} \Delta_{1}^{-1}(a+b+c+d+e) \\
& -\frac{1}{4} \Delta^{-1} a_{x} a_{3 x} \Delta_{3}^{-1}(b+c+e)+\frac{1}{6} \Delta^{-1} a_{x} G_{11} \Delta_{1}^{-1}\left(a+b+c+\frac{3}{4} d+\frac{3}{4} e\right) \\
& -\frac{1}{8} \Delta^{-1} a_{x} G_{13} \Delta_{3}^{-1}\left(\frac{4}{3} b+c+e\right)-\frac{1}{4} \Delta^{-1} a_{y} a_{1 x} \Delta_{1}^{-1}(a+b+c+d e) \\
& +\frac{1}{4} \Delta^{-1} a_{y} a_{2 x} \Delta_{2}^{-1}(a+d)-\frac{1}{6} \Delta^{-1} a_{y} G_{11} \Delta_{1}^{-1}\left(a+b+c+\frac{3}{4} d+\frac{3}{4} e\right) \\
& +\frac{1}{6} \Delta^{-1} a_{y} G_{12} \Delta_{2}^{-1}\left(a+\frac{3}{4} d\right) \\
& +\frac{1}{4} V(0,0) \ln V(0,0)\left[\Delta^{-1} a_{x} a_{1 x} \Delta_{1}^{-1}-\Delta^{-1} a_{y} a_{1 x} \Delta_{1}^{-1}+\Delta^{-1} a_{y} a_{2 x} \Delta_{2}^{-1}\right] \\
& -\frac{1}{4} V(1,0) \ln V(1,0)\left[G_{2} \Delta^{-1} a_{3 x} \Delta_{3}^{-1}+\Delta^{-1} a_{x} a_{3 x} \Delta_{3}^{-1}+4 \Delta^{-1} a_{y} a_{2 x} \Delta_{2}^{-1}\right. \\
& \left.+\Delta^{-1} a_{y} G_{12} \Delta_{2}^{-1}\right] \\
& +\frac{1}{4} V(1,1) \ln V(1,1)\left[G_{2} \Delta^{-1} a_{3 x} \Delta_{3}^{-1}+G_{2} \Delta^{-1} G_{13} \Delta_{3}^{-1}-\Delta^{-1} a_{x} a_{1 x} \Delta_{1}^{-1}\right. \\
& +\Delta^{-1} a_{x} a_{3 x} \Delta_{3}^{-1} \\
& \left.-\Delta^{-1} a_{x} G_{11} \Delta_{1}^{-1}+\Delta^{-1} a_{x} G_{13} \Delta_{3}^{-1} \Delta^{-1} a_{y} a_{1 x} \Delta_{1}^{-1}+\Delta^{-1} a_{y} G_{11} \Delta_{1}^{-1}\right] \text {. }
\end{aligned}
$$

Here, the Gram determinants are given by

$$
\begin{aligned}
a_{x} & =2 d b-c e, a_{y}=2 e a-d c, \quad a_{1 x}=\frac{d+e}{2}, a_{2 x}=\frac{d}{2}, a_{3 x}=\frac{c+e}{2}, \\
\Delta & =f G_{2}-\left(b d^{2}-c d e+a e^{2}\right), \\
\Delta_{1} & =f G_{11}-\frac{(d+e)^{2}}{4}, \quad \Delta_{2}=f G_{12}-\frac{d^{2}}{4}, \quad \Delta_{3}=(a+d+f) G_{13}-\frac{(c+e)^{2}}{4}, \\
G_{2} & =4 a b-c^{2}, \quad G_{11}=a+b+c, \quad G_{12}=a, \quad G_{13}=b,
\end{aligned}
$$

Another example is given by the IR-divergent $C_{0^{-}}$functions. One can prove that $B=0$ for

$$
a=b=f=m^{2}, \quad c=e=s-2 m^{2}, \quad d=-2 m^{2} .
$$


As a consequence we have

$$
\left[1+P_{x} \frac{\partial}{\partial_{x}}+P_{y} \frac{\partial}{\partial_{y}}\right] V^{\mu+1}(x, y)=0, \quad P_{x ; y}=\frac{1-x ;-y}{2(\mu+1)} .
$$

This relation we use to write

$$
\begin{aligned}
& \int_{0}^{1} d x \int_{0}^{x} d y V^{-1-\varepsilon / e}(x, y)=\frac{1}{\varepsilon} \int_{0}^{1} d x V^{-1-\varepsilon / e}(x, x), \\
& {\left[1+\frac{1}{2(\mu+1)}\left(\frac{1}{2}-x\right) \partial_{x}\right] V^{1+\mu}(x, x) }=-\frac{1}{4}\left(s-4 m^{2}\right) V^{\mu}(x, x) \\
& C_{0}^{\mathrm{IR}}= \frac{1}{s-4 m^{2}}\left\{\left(-\frac{1}{2}+\frac{1}{\varepsilon}\right)\left[1-\frac{1}{4} \ln V(0,0)-\frac{1}{4} \ln V(1,1)\right]\right. \\
&\left.+\left(-\frac{3}{4}+\frac{1}{2 \varepsilon}\right) \int_{0}^{1} d x \ln V(x, x)\right\}
\end{aligned}
$$

Another example is the massless pentagon. Here (but also for the massive one) one can prove that

$$
\begin{gathered}
E_{0}=\int_{0}^{1} d x_{1} \int_{0}^{1-x_{1}} d x_{2} \int_{0}^{1-x_{1}-x_{2}} d x_{3} \int_{0}^{1-x_{1}-x_{2}-x_{3}} d x_{4}\left[x^{t} H^{-1} x+2 K^{t} x\right]^{-3-\varepsilon / 2}, \\
H=\frac{1}{2}\left(\begin{array}{cccc}
0 & -s_{51} & s_{12}-s_{34} & s_{45} \\
- & -2 s_{51} & -s_{34}-s_{51} & s_{23}-s_{51} \\
- & - & -2 s_{34} & -s_{34} \\
- & - & - & 0
\end{array}\right) \\
K_{1}=0, \quad K_{2}=\frac{1}{2} s_{51}, \quad K_{3}=\frac{1}{2} s_{34}, \quad K_{4}=0, \quad s_{i j}=-\left(p_{i}+p_{j}\right)^{2} .
\end{gathered}
$$

Now we raise the exponent and integrate by parts with $B=1 / 16 s_{12} s_{23} s_{34} s_{45} s_{51}$.

In moving to multi-loop we recall that any diagram $G$ with $N_{L}$ legs and $n_{l}$ loops is representable as

$$
\begin{aligned}
G & =(-1)_{L}^{N}\left(\frac{i}{\pi^{n / 2}}\right)^{n_{l}} \Gamma\left(N_{L}-\frac{n}{2} n_{l}\right) \int \frac{d z_{G} \delta\left(1-z_{G}\right)}{U^{n / 2}(V-i \varepsilon)^{N_{L}-n n_{l} / 2}}, \\
V & =\sum_{i} m_{i}^{2} z_{i}-\sum_{i} q_{i}^{2} z_{i}-\frac{1}{U} \sum_{i j} B_{i j} q_{i} \cdot q_{j} z_{i} z_{j} \\
U & =\sum_{T} \prod_{z_{i} \in T} z_{i}=\operatorname{det}\left(U_{r s}\right), \quad U_{r s}=\sum_{i} z_{i} \eta_{i r} \eta_{i s} .
\end{aligned}
$$


$\eta_{i s}$ is the projection of line $i$ along the loop $s . T$ is a co-tree. Note that UV-singularities come from $U$ so that, for finite diagrams, one should raise only the factor

$$
\tilde{V}=U\left[\sum_{i} m_{i}^{2} z_{i}-\sum_{i} q_{i}^{2} z_{i}\right]-\sum_{i j} B_{i j} q_{i} \cdot q_{j} z_{i} z_{j}
$$

There are several comments. For two-loops $U$ is quadratic and $\tilde{V}$ is cubic, so that we have to construct a Bernstein functional relation for a quintic or higher polynomial. Try to do it naively in FORM and you will be told 'Input expansion buffer overflow. Try something else.'

For $N_{L}=3,4$, we have $\tilde{V}$ to a positive power and it is enough to regularize $G$ with some $K_{S}$ operation $(S \in G)$. For $N_{L}>5$, we have $\tilde{V}$ to a negative power, $U$ to a positive power (no UV divergency), so we could raise a cubic. For self-energies at $q^{2}=0$ it is easy. Consider, for example, $n_{l}=2, N_{L}=3$, perform a projective transformation

$$
z_{i}=A_{i} u_{i} / \sum_{j} A_{j} u_{j}, \quad A_{i}=\frac{1}{m_{i}^{2}}
$$

to obtain $\tilde{V}=-U$ quadratic and,

$$
\begin{aligned}
\tilde{V} & =-x y\left(\frac{1}{m_{1}^{2} m_{2}^{2}}-\frac{1}{m_{1}^{2} m_{3}^{2}}+\frac{1}{m_{2}^{2} m_{3}^{2}}\right)-x \frac{1}{m_{1}^{2} m_{2}^{2}} \\
& +x^{2} \frac{1}{m_{1}^{2} m_{2}^{2}}+y\left(\frac{1}{m_{1}^{2} m_{2}^{2}}-\frac{1}{m_{1}^{2} m_{3}^{2}}\right)+y^{2} \frac{1}{m_{2}^{2} m_{3}^{2}}
\end{aligned}
$$

The crucial point being that we know how to raise a quadratic. Finally, $N_{L}=2\left(n_{l}+1\right)$ is nice, a single polynomial of degree $n_{l}+1$. Of course, vacuum diagrams are also easy. At two-loop level we have

$$
\begin{aligned}
G_{2}^{\mathrm{vac}} & =(4 \pi)^{\varepsilon-4} \Gamma(\varepsilon-1)\left(K^{t} H^{-1} K\right)^{-2} \int_{0}^{1} d x \int_{0}^{x} d y \\
& \times\left[1-\frac{\left(x+K^{t} H^{-1}\right) \partial}{\varepsilon-2}\right]\left[1-\frac{\left(x+K^{t} H^{-1}\right) \partial}{\varepsilon}\right] U^{\varepsilon / 2}(x, y),
\end{aligned}
$$

where $U$ has been presented above. The same is, obviously true, for tadpoles. What we are still missing is a real multi-loop example,

Additional problems are represented by the fact that $\tilde{V}$ is an highly incomplete polynomial, few non-zero coefficients. $\mathcal{P}$ can be found via a direct study of linear systems of $n$ equations $(n \gg 100)$ in $m$ variables and very often the matrix of coefficients has rank $<\mathrm{n}$, so the system is generally impossible and, we need to go to higher $n, m$, i.e. beyond $n_{\min }=\min \{n<m\}$ (try one-loop master formulae with $K=L=0$ !) and your algebraic manipulation will exhaust your computer before you find a solution. 
A simple counting shows the following situation: given

$$
\mathcal{P}=P_{n}+P_{n+1}^{i} \partial_{i}+P_{n+2}^{i j} \partial_{i} \partial_{j}+\ldots
$$

four variables and a cubic $V$ require $n_{\text {eq }}=126(330,715)$ and $n_{\text {var }}=155(415,871)$ for $n=2(1,0)$ and first(second, third) derivatives. Simple examples already show that, for realistic polynomials $V$, one has to go beyond second order in derivatives.

The relation between $B=0$ and Landau singularities is still to be examined, although $B=0$ contains all singularities of $G$, physical and spurious ones. $L-$ $K^{t} H^{-1} K$ (or $B$ in general) is not a Gram determinant but its spurious zeros, if any, are a vexation, as much as those of Gram determinants in the standard reduction procedure. For $C_{0}\left(-m_{a}^{2},-m_{b}^{2},-s ; m_{b}, M, m_{a}\right)$ we obtain

$$
\begin{aligned}
G_{11} & =-\frac{1}{4} \lambda\left(s, m_{a}^{2}, m_{b}^{2}\right), \quad G_{12}=-\frac{1}{4} \lambda\left(M^{2}, m_{a}^{2}, m_{b}^{2}\right), \quad G_{13}=-\frac{1}{4} \lambda\left(M^{2}, m_{a}^{2}, m_{b}^{2}\right), \\
G_{2} & =\left[\left(m_{a}^{2}-m_{b}^{2}\right)^{2}-M^{2} s\right]\left[s+M^{2}-2\left(m_{a}^{2}+m_{b}^{2}\right)\right] .
\end{aligned}
$$

\section{References}

[1] D. Bardin, M. Grunewald and G. Passarino, hep-ph/9902452.

[2] G. Montagna, F. Piccinini, O. Nicrosini, G. Passarino and R. Pittau, Comput. Phys. Commun. 76 (1993) 328.

[3] G. Montagna, F. Piccinini, O. Nicrosini, G. Passarino and R. Pittau, Nucl. Phys. B401 (1993) 3.

[4] D. Bardin, P. Christova, M. Jack, L. Kalinovskaya, A. Olchevski, S. Riemann and T. Riemann, hep-ph/9908433.

[5] S. Jadach, B. F. Ward and Z. Was, Comput. Phys. Commun. 130 (2000) 260 hep-ph/9912214.

[6] LEP EWWG/LS 2000-01.

[7] S. Jadach, W. Placzek, E. Richter-Was, B. F. Ward and Z. Was, Comput. Phys. Commun. 102 (1997) 229.

[8] A. Denner, S. Dittmaier, M. Roth and D. Wackeroth, Nucl. Phys. B587 (2000) 67 hep-ph/0006307.

[9] S. Jadach, W. Placzek, M. Skrzypek, B. F. Ward and Z. Was, hep-ph/0007012.

[10] M. W. Grunewald et al., hep-ph/0005309. 
[11] W. Beenakker et al., Nucl. Phys. B500 (1997) 255 hep-ph/9612260.

[12] G. Passarino, Nucl. Phys. B578 (2000) 3 hep-ph/0001212].

[13] G. Passarino, Nucl. Phys. B574 (2000) 451 hep-ph/9911482].

[14] C. Chahine, Phys. Rev. D18 (1978) 4617.

[15] F. V. Tkachov, Nucl. Inst. \& Meth. A389 (1997)309.

[16] D. Y. Bardin, L. V. Kalinovskaya and F. V. Tkachov, hep-ph/0012209. 